\title{
On the Surgery Theory for Filtered Manifolds
}

\author{
Alberto Cavicchioli $^{1, *}$, Friedrich Hegenbarth ${ }^{2}$, Yuri Muranov ${ }^{3}$, Fulvia Spaggiari ${ }^{1}$ \\ ${ }^{1}$ Dipartimento di Scienze Fisiche, Informatiche e Matematiche \\ Università di Modena e Reggio Emilia, Via Campi 213/B, 41100 Modena, Italy \\ ${ }^{2}$ Dipartimento di Matematica, Università di Milano, Via Saldini n. 50, 20133 Milano, Italy \\ ${ }^{3}$ Department of Mathematics, Grodno State University, Ozheshko str. 22, 230023 Grodno, Belarus \\ *alberto.cavicchioli@unimore.it \\ Copyright (C)2013 Horizon Research Publishing All rights reserved.
}

\begin{abstract}
In this paper we describe some relations between various structure sets which arise naturally for a Browder-Livesay filtration of a closed topological manifold. We use the algebraic surgery theory of Ranicki for realizing the surgery groups and natural maps on the spectrum level. We obtain also new relations between Browder-Quinn surgery obstruction groups and structure sets. Finally we illustrate several examples and applications.
\end{abstract}

Keywords Surgery on manifolds, Browder-Livesay filtration, Browder-Quinn surgery obstruction groups, Surgery on stratified manifolds, Splitting obstruction groups, Surgery exact sequence, Structure sets, Normal invariants

Mathematics Subject Classification (2000): Primary 57R67, 19J25 Secondary 55T99, 58A35, 18F 25

\section{Introduction.}

Let $X^{n}$ be a closed connected $n$-dimensional topological manifold with fundamental group $\pi=\pi_{1}(X)$. We suppose that the group $\pi$ is equipped with an orientation map $w: \pi=\pi_{1}(X) \rightarrow\{ \pm 1\}$ which coincides with the Stiefel-Whitney character. An $s$-triangulation of a manifold $X^{n}$ is a simple homotopy equivalence $f: M \rightarrow X$, where $M$ is a closed $n$-dimensional topological manifold. Two $s$-triangulations $f_{i}: M_{i} \rightarrow X, i=1,2$, are called equivalent if there is a homotopy commutative diagram

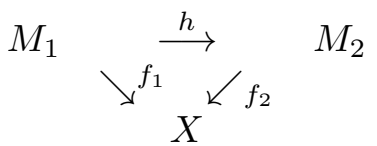

where $h$ is an orientation preserving homeomorphism. Let $\mathcal{S}^{s}(X)$ be the set of equivalence classes of $s$-triangulations of the manifold $X$. For $n \geq 5$, the structure 
set $\mathcal{S}^{s}(X)$ fits into the Sullivan-Novikov-Wall surgery exact sequence (see [19], [20], and $[22])$

$$
\cdots \rightarrow L_{n+1}(\pi) \rightarrow \mathcal{S}^{s}(X) \rightarrow[X, G / T O P] \stackrel{\sigma}{\rightarrow} L_{n}(\pi) \rightarrow \cdots
$$

This sequence is the main tool for computing the structure set $\mathcal{S}^{s}(X)$. The set $[X, G / T O P]$ coincides with the set of normal invariants $\mathcal{T}(X)$ which is formed by the normal bordism classes of normal maps into the manifold $X$. Given a normal map (also called a $t$-triangulation of $X)(f, b) \in \mathcal{T}(X)$, the map $\sigma$ assigns a surgery obstruction $\sigma(f) \in L_{n}(\pi)$. This is trivial if and only if there is a simple homotopy equivalence in the normal bordism class of the map $(f, b)$.

Let $\left(X^{n}, Y^{n-q}, \xi\right)$ be a topological codimension $q$ manifold pair [20, p.570], where $\xi$ is a topological normal block bundle. We assume that $n-q \geq 5$. A $t$-triangulation

$$
((f, b),(g, c)):(M, N) \rightarrow(X, Y)
$$

of a manifold pair $\left(X^{n}, Y^{n-q}, \xi\right)$ is given by a normal map $(f, b): M \rightarrow X$, which is transversal to $Y, N=f^{-1}(Y)$, and the following properties are satisfied [20]:

(i) $(M, N)$ is a topological manifold pair with a topological normal block bundle

$$
\nu: N \stackrel{\left.f\right|_{N}}{\rightarrow} Y \stackrel{\xi}{\rightarrow} \widetilde{\operatorname{BTOP}}(q)
$$

(ii) the restriction $\left.(f, b)\right|_{N}$ coincides with the normal map $(g, c): N \rightarrow Y$;

(iii) the restriction

$$
\left.(f, b)\right|_{P}:(P, S(\nu)) \rightarrow(Z, S(\xi))
$$

is a normal map, where

$$
P=\overline{M \backslash D(\nu)} \quad \text { and } \quad Z=\overline{X \backslash D(\xi)} ;
$$

(iv) the restriction $\left.(f, b)\right|_{S(\nu)}$ coincides with the transfer map

$$
\left.(g, c)^{!}\right|_{S(\nu)}: S(\nu) \rightarrow S(\xi)
$$

and $(f, b)=\left.(g, c)^{!} \cup(f, b)\right|_{P}$.

Any normal map $(f, b)$ defines a $t$-triangulation of the manifold pair $(X, Y, \xi)$, and the set $\mathcal{T}(X, Y)$ of concordance classes of $t$-triangulations of $(X, Y, \xi)$ coincides with $\mathcal{T}(X)$ [20, Proposition 7.2.3].

A simple homotopy equivalence $f: M \rightarrow X$ splits along the submanifold $Y$ if $f$ is homotopic to a map $g: M \rightarrow X$ which is transversal to $Y$ with a transversal preimage $N=g^{-1}(Y)$ and whose restrictions

$$
\left.g\right|_{N}: N \rightarrow Y,\left.\quad g\right|_{(M \backslash N)}: M \backslash N \rightarrow X \backslash Y,\left.\quad g\right|_{P}:(P, S(\nu)) \rightarrow(Z, S(\xi))
$$

are simple homotopy equivalences (see [19], [20, §7.2] and [22]). The splitting obstruction groups $L S_{*}(F)$ are defined (see the quoted papers). If $f: M \rightarrow X$ is a simple homotopy equivalence, then an obstruction to find in its homotopy class a map transversal to $Y$ with properties (1.3) lies in the splitting obstruction group 
$L S_{n-q}(F)$. The $L S_{*}$-groups depend only on $n-q(\bmod 4)$ and on the pushout square

$$
F=\left(\begin{array}{ccc}
\pi_{1}(\partial U) & \rightarrow & \pi_{1}(X \backslash Y) \\
\downarrow & & \downarrow \\
\pi_{1}(U) & \rightarrow & \pi_{1}(X)
\end{array}\right)
$$

of fundamental groups with orientations, where $U$ is a tubular neighborhood of $Y$ in $X$. For a manifold pair $(X, Y, \xi)$ the surgery obstruction groups $L P_{n-q}(F)$ are also defined (see [19], [20], and [22]), and they depend only on $n-q(\bmod 4)$ and on the square $F$.

There are the assigning obstruction maps

$$
\mathcal{S}^{s}(X) \rightarrow L S_{n-q}(F) \text { and } \mathcal{T}(X) \rightarrow L P_{n-q}(F)
$$

and the forgetful maps [20]

$$
\mathcal{S}^{s}(X, Y, \xi) \rightarrow \mathcal{S}^{s}(X), \quad \mathcal{S}^{s}(X, Y, \xi) \rightarrow \mathcal{T}(X), \text { and } \mathcal{S}^{s}(X, Y, \xi) \rightarrow \mathcal{S}^{s}(Y)
$$

Let $(f, b): M \rightarrow X$ be a normal map which is transversal to a submanifold $Y$, with $N=f^{-1}(Y)$, and let $\left.f\right|_{N}: N \rightarrow Y$ be a simple homotopy equivalence.

Two such maps $f_{i}: M_{i} \rightarrow X(i=1,2)$ are said to be equivalent [2] if there exists a normal bordism map with the following properties:

i) $\partial W=M_{1} \cup M_{2}$ and $\left.F\right|_{M_{i}}=f_{i}(i=1,2)$;

ii) the map $F$ is transversal to $Y$ with $F^{-1}(Y)=V$, and $\partial V=N_{1} \cup N_{2}$;

iii) the restriction $\left.F\right|_{V}$ is an $s$-cobordism between $\left.F\right|_{N_{1}}=f_{1}$ and $\left.F\right|_{N_{2}}=f_{2}$.

We denote by $\mathcal{N S}^{s}(X, Y)$ (see [2]) the set of the correspondent equivalence classes. Then we have the following natural maps

$$
\begin{gathered}
\mathcal{N S}^{s}(X, Y) \rightarrow \mathcal{T}(X), \quad \mathcal{N S}^{s}(X, Y) \rightarrow \mathcal{S}^{s}(Y), \\
\mathcal{S}^{s}(X, Y, \xi) \rightarrow \mathcal{N S}^{s}(X, Y), \quad L_{n-q+1}\left(\pi_{1}(Y)\right) \stackrel{\lambda}{\rightarrow} \mathcal{N} \mathcal{S}^{s}(X, Y) .
\end{gathered}
$$

There are many relations between the above-defined structure sets (see [1], [2], [8], [9], [13], [17], [18], and [20, §7.2]).

The surgery theory for filtered manifolds is very close to the classical surgery theory (see [3], [6], [17], and [24]). Let $\mathcal{X}$ be a filtration

$$
X_{k} \subset X_{k-1} \subset \cdots \subset X_{2} \subset X_{1} \subset X_{0}=X \quad\left(\operatorname{dim} X_{j}=n_{j}\right)
$$

of a closed manifold $X$ by means of closed submanifolds. We assume that $\operatorname{dim} X_{k}=$ $n_{k} \geq 5$, and that every pair of manifolds of the filtration is a closed manifold pair.

The filtration $\mathcal{X}$ in (1.8) is a stratified manifold in the sense of Browder-Quinn. Doing a topological normal map $f: M \rightarrow X$ topologically transversal to the submanifolds $X_{i}$ gives a $t$-triangulation of the filtration $\mathcal{X}$ (see [3], [6], [17], and [24]) with $f^{-1}\left(X_{i}\right)=M_{i}$. A $t$-triangulation $(f, b): M \rightarrow X$ is an $s$-triangulation of the filtration $\mathcal{X}$ if all the restricted normal maps of pairs

$$
\left.f\right|_{\left(M_{j}, M_{l}\right)}:\left(M_{j}, M_{l}\right) \rightarrow\left(X_{j}, X_{l}\right), \quad 0 \leq j<l \leq k,
$$


are $s$-triangulations. The group $L_{n_{k}}^{B Q}(\mathcal{X})$ is the group of obstructions to find an $s$-triangulation of the filtration $\mathcal{X}$ in the class of the normal bordism of a $t$ triangulation of $\mathcal{X}$. The set of concordance classes of $s$-triangulations of $\mathcal{X}$ is denoted by $\mathcal{S}^{s}(\mathcal{X})$. It fits into the exact sequence (see [3], [6], [17], and [24])

$$
\cdots \rightarrow L_{n+1}^{B Q}(\mathcal{X}) \rightarrow \mathcal{S}^{s}(\mathcal{X}) \rightarrow \mathcal{T}(X) \rightarrow L_{n}^{B Q}(\mathcal{X})
$$

which generalizes the surgery exact sequence in (1.1) to the case of stratified manifolds. Some relations between various structure sets for the filtration in (1.8) were obtained in [3], [13], and [17].

Denote by $F_{i}(0 \leq i \leq k-1)$ the square of fundamental groups in the splitting problem for the manifold pair $\left(X_{i}, X_{i+1}\right)$ fitting into filtration (1.8). We shall call this pair of manifolds a Browder-Livesay pair if $X_{i+1}$ is a one-sided submanifold of $X_{i}$, and the inclusion $X_{i+1} \rightarrow X_{i}$ induces an isomorphism of the fundamental groups. In this case, the group of obstructions for the splitting problem is denoted by $L N_{*}\left(\pi_{1}\left(X_{i} \backslash X_{i+1}\right) \rightarrow \pi_{1}\left(X_{i}\right)\right)$, and it is called the Browder-Livesay group (see [5], [7], [11], [12], [14], [16], [20], and [22]). A filtration as in (1.8), for which every pair of submanifolds $\left(X_{i}, X_{i+1}\right)$ is a Browder-Livesay pair, is called a BrowderLivesay filtration (see [3] and [17]). The notion of Browder-Livesay filtration is very useful to investigate assembly maps, iterated Browder-Livesay invariants, surgery spectral sequences, natural maps in surgery theory, and actions of 2-groups on manifolds (see [1], [5], [7], [9], [11], [12], [14], [16], [20], and [22]).

For a Browder-Livesay filtration $\mathcal{X}(1.8)$ and for a simple homotopy equivalence $f: M \rightarrow X$ there is an obstruction in the group $L S F_{n-k}(\mathcal{X})$ to find an $s$ triangulation of $\mathcal{X}$ in the homotopy class of the map $f$ [3]. The groups $L S F_{*}(\mathcal{X})$ are a natural generalization of the splitting obstruction groups $L S_{*}$ and relate with other surgery obstruction groups and structure sets for $\mathcal{X}$ by various braids of exact sequences [3]. The main connection is given by the following braid of exact sequences

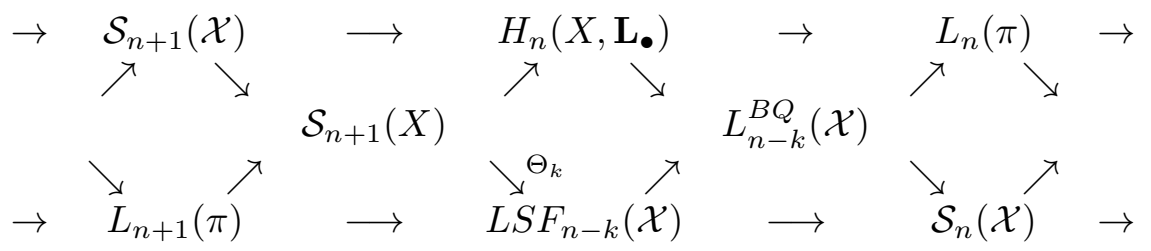

where $\pi=\pi_{1}(X)$. In this paper we describe new relations between structure sets and various obstruction groups which arise for a Browder-Livesay filtration. From now on we shall consider only Browder-Livesay filtrations, but the results without difficulties can be transferred to the general case. We use the algebraic surgery theory of Ranicki for realizing the surgery groups and natural maps on the spectrum level. Finally, we discuss several examples and applications.

\section{Surgery spectra.}

First we recall some necessary results about the realization of surgery obstruction groups, structure sets, and natural maps on the spectrum level (see [1], [2], [8], [9], [10], [11], [15], [16], [17], [18], [18], [20], and [24]). We use standard notations for spectra which realize various surgery and splitting obstruction groups and 
structure sets. Thus we have spectra $\mathbb{L}(\pi), \mathbb{L} S(F), \mathbb{L} P(F), \mathbb{L} N(\pi \rightarrow G), \mathbb{L}^{B Q}(\mathcal{F})$, and $\mathbb{L} S F(\mathcal{F})$, that realize the corresponding obstruction groups. For example, $\pi_{i}(\mathbb{L} S(F))=L S_{i}(F)$, and similarly for the other spectra. For any $\Omega$-spectrum $\mathbb{A}$, $\Omega \mathbb{A}$ is the corresponding loop spectrum, and $\Omega^{-1} \mathbb{A}$ represents the de-looping spectrum. Many natural maps, as the surgery transfer maps and the induced maps, are also realized on the spectra level, as well as, the corresponding relative obstruction groups. The map $\sigma$ in (1.1) is realized by the cofibration of spectra

$$
X_{+} \wedge \mathbf{L}_{\bullet} \rightarrow \mathbb{L}(\pi)
$$

with cofiber $\mathbb{S}(X)$ such that $\mathcal{S}_{n}(X)=\pi_{n}(\mathbb{S}(X))$, and there is an isomorphism of structure sets

$$
\mathcal{S}^{s}(X)=\mathcal{S}_{n+1}(X), \mathcal{T}(X)=H_{n}\left(X, \mathbf{L}_{\bullet}\right)(\operatorname{dim} X=n \geq 5) .
$$

Surgery exact sequence (1.1) is isomorphic to the left part starting from $L_{n}\left(\pi_{1}(X)\right)$ of homotopy long exact sequence of cofibration (2.1). We have similar situations for other structure sets and natural maps. For a closed manifold pair $\left(X^{n}, Y^{n-q}\right)$ the maps from (1.5), (1.6) and (1.7) are realized by the maps of spectra

$$
\begin{gathered}
\Omega^{q+1} \mathbb{S}(X) \rightarrow \mathbb{L} S(F), \Omega^{q}\left(X_{+} \wedge \mathbf{L}_{\bullet}\right) \rightarrow \mathbb{L} P(F), \mathbb{S}(X, Y) \rightarrow \mathbb{S}(X), \\
\Omega \mathbb{S}(X, Y) \rightarrow X_{+} \wedge \mathbf{L}_{\bullet}, \Omega^{q} \mathbb{S}(X, Y) \rightarrow \mathbb{S}(Y), \Omega \mathbb{S}(X, Y) \rightarrow \mathbb{N} S(X, Y), \\
\Omega^{k} \mathbb{N} S(X, Y) \rightarrow \mathbb{S}(Y), \Omega \mathbb{S}(X, Y) \rightarrow \mathbb{N} S(X, Y), \mathbb{L}\left(\pi_{1}(Y)\right) \rightarrow \Omega^{k} \mathbb{N} S(X, Y),
\end{gathered}
$$

where $\mathbb{S}(X, Y)=\mathbb{S}(X, Y, \xi), k=q-1$. For the homotopy groups of spectra that realize structure sets, we shall use the following notations

$$
\pi_{i}(\mathbb{S}(X, Y))=\mathcal{S}_{i}(X, Y), \pi_{i}(\mathbb{N} S(X, Y))=\mathcal{N} S_{i}(X, Y), \pi_{i}(\mathbb{S}(Y))=\mathcal{S}_{i}(Y)
$$

and we have isomorphisms

$$
\mathcal{S}_{n+1}(X, Y)=\mathcal{S}^{s}(X, Y), \mathcal{N} S_{n}(X, Y)=\mathcal{N} S^{s}(X, Y), \mathcal{S}_{n-q+1}(Y)=S^{s}(Y) .
$$

Let us consider a Browder-Livesay filtration $\mathcal{X}$ as in (1.8). For $0 \leq l<j \leq k$, a restricted filtration

$$
X_{j} \subset X_{j-1} \subset \cdots \subset X_{l}
$$

is well-defined, and we denote it by $\mathcal{X}_{j}^{l}$. Let $\mathcal{X}_{k}^{l}=\mathcal{X}^{l}$ and $\mathcal{X}_{j}^{0}=\mathcal{X}_{j}$. The filtration $\mathcal{X}$ yields a filtration of manifolds with boundary

$$
\left(X_{k-1} \backslash X_{k}, \partial\left(X_{k-1} \backslash X_{k}\right)\right) \subset \cdots \subset\left(X_{0} \backslash X_{k}, \partial\left(X_{0} \backslash X_{k}\right)\right)
$$

which is a $\mathcal{C}$-stratified manifold with boundary (see [6], [17], and [24]). Let us denote the filtration in $(2.4)$ by $\overline{\mathcal{X}}$. For the spectrum $\mathbb{L}^{B Q}(\mathcal{X})$ (see [3] and [24]) we have isomorphisms

$$
\pi_{i}\left(\mathbb{L}^{B Q}(\mathcal{X})\right)=L_{i}^{B Q}(\mathcal{X})
$$


The subscript $*$ in the obstruction group $L_{*}^{B Q}(\mathcal{X})$ for filtration (1.8) equals the dimension $n-k$ of the smallest manifold of the filtration.

Remark that the filtration $\overline{\mathcal{X}}$ in $(2.4)$ of manifolds with boundary defines a filtration of closed manifolds

$$
\partial\left(X_{k-1} \backslash X_{k}\right) \subset \partial\left(X_{k-2} \backslash X_{k}\right) \subset \cdots \subset \partial\left(X_{1} \backslash X_{k}\right) \subset \partial\left(X_{0} \backslash X_{k}\right)
$$

which we denote by $\partial \overline{\mathcal{X}}$. It follows from the definition that each one of the filtrations $\partial \overline{\mathcal{X}}$ and $\overline{\mathcal{X}}$ contains $k$ spaces. The inductive definition of the spectra $\mathbb{L}^{B Q}$ is given in [24]. The spectra $\mathbb{L}^{B Q}\left(\mathcal{X}_{j}\right)$ fit into the following cofibration sequence

$$
\cdots \rightarrow \mathbb{L}^{B Q}\left(\mathcal{X}_{j}\right) \rightarrow \mathbb{L}\left(G_{j}\right) \rightarrow \mathbb{L}^{B Q}\left(\overline{\mathcal{X}_{j}}\right) \rightarrow \Omega^{-1} \mathbb{L}^{B Q}\left(\mathcal{X}_{j}\right) \rightarrow \cdots
$$

as follows immediately from the definition. For $1 \leq j \leq k$ we denote by $\mathcal{Y}_{j}=\mathcal{X}_{j}^{1}$ the subfiltration

$$
X_{j} \subset X_{j-1} \subset \cdots \subset X_{2} \subset X_{1}
$$

of the filtration $\mathcal{X}$. Then the filtrations $\overline{\mathcal{Y}_{j}}$ and $\partial \overline{\mathcal{Y}_{j}}$ are defined as before. Let $\mathcal{Y}=\mathcal{Y}_{k}$. Note also that the braid of exact sequences in (1.10) is also realized by the pull-back square of spectra [3]

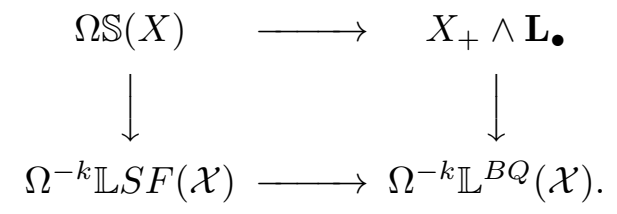

We observe that in (2.9) the cofiber of horizontal maps is $\mathbb{L}\left(\pi_{1}(X)\right)$ while the cofiber of the vertical maps is $\mathbb{S}(\mathcal{X})$ with $\pi_{i}(\mathbb{S}(\mathcal{X}))=\mathcal{S}_{i}(\mathcal{X})$ and $\mathcal{S}_{n+1}(\mathcal{X})=S^{s}(\mathcal{X})$.

\section{Structure sets of a Browder-Livesay filtration.}

Here we use notations from the previous sections. For a Browder-Livesay filtration $\mathcal{X}$ in (1.8) we have $\operatorname{dim} X_{i}=n-i(0 \leq i \leq k)$. Let $G_{i}=\pi_{1}\left(X_{i}\right)(0 \leq i \leq k)$ and $\rho_{i}=\pi_{1}\left(X_{i} \backslash X_{i+1}\right)(0 \leq i \leq k-1)$.

Theorem 3.1. There exists a pullback square of spectra

$$
\begin{array}{cccc}
\mathbb{N} S\left(X, X_{k}\right) & \rightarrow & \Omega^{-k+1} \mathbb{L}^{B Q}(\overline{\mathcal{X}}) \\
\downarrow & & \downarrow \\
X_{+} \wedge \mathbf{L}_{\bullet} & \rightarrow & \Omega^{-k} \mathbb{L}^{B Q}(\mathcal{X})
\end{array}
$$

in which the cofibres of the vertical maps are $\Omega^{-k} \mathbb{L}\left(G_{k}\right)$, and the cofibres of the horizontal maps are $\mathbb{S}(\mathcal{X})$.

Square (3.1) defines a braid of exact sequences

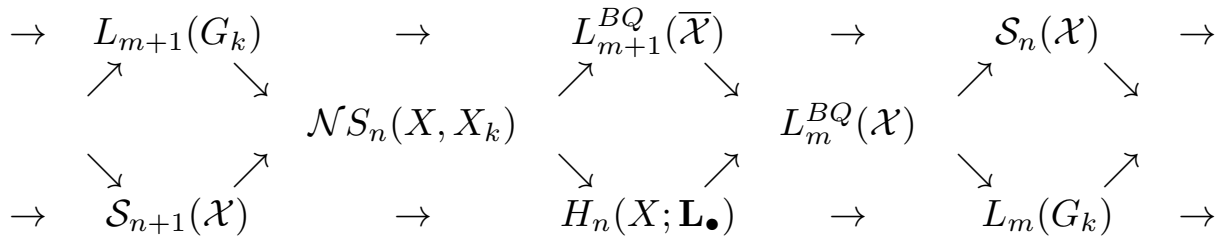


where $m=n-k$.

Proof. We have a homotopy commutative square of spectra

$$
\begin{array}{cccc}
X_{+} \wedge \mathbf{L} \bullet & \rightarrow & \Omega^{-k} \mathbb{L}^{B Q}(\mathcal{X}) \\
\downarrow & & \downarrow \\
\Omega^{-k} \mathbb{L}\left(G_{k}\right) & \rightarrow & \Omega^{-k} \mathbb{L}\left(G_{k}\right)
\end{array}
$$

in which the upper horizontal map is the map from (2.9), the left vertical map was constructed on the spectra level in [2], and the right vertical map is the natural forgetful map fitting in (2.7). It follows immediately from [2] and [24] that Diagram (3.3) is homotopy commutative. The fiber of the left vertical map in (3.3) is $\mathbb{N} S\left(X, X_{k}\right)$ by [2], and the fiber of the right vertical map in (3.3) is $\Omega^{-k+1} \mathbb{L}^{B Q}(\overline{\mathcal{X}})$ as follows from (2.7). We consider Diagram (3.3) in which the horizontal maps induce a map of fibres of the vertical maps (see [23]). Thus we obtain the pullback square in (3.1). Now the homotopy long exact sequences of the maps from (3.1) generate the braid of exact sequences in (3.2).

Proposition 3.2. Let $\mathcal{X}$ be a Browder-Livesay filtration as in (1.8). For $0 \leq i<$ $j \leq k-1$ we have pullback squares of spectra

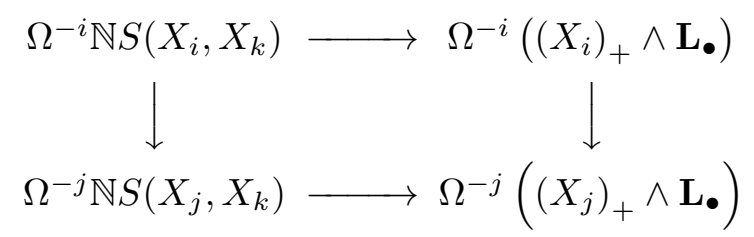

and

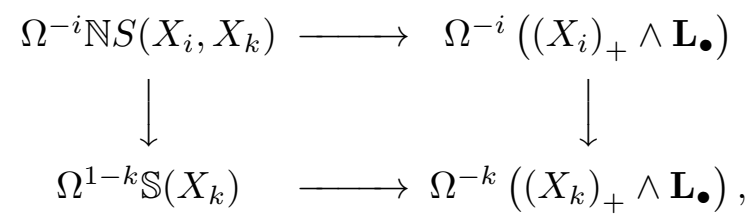

where the cofibers of all horizontal maps are naturally homotopy equivalent to $\Omega^{-k} \mathbb{L}\left(G_{k}\right)$, the fibers of vertical maps in (3.4) are naturally homotopy equivalent to $\Omega^{-i}\left(\left(X_{i} \backslash X_{j}\right)_{+} \wedge \mathbf{L}_{\bullet}\right)$, and the fibers of the vertical maps in (3.5) are naturally homotopy equivalent to $\Omega^{-i}\left(\left(X_{i} \backslash X_{k}\right)_{+} \wedge \mathbf{L}_{\bullet}\right)$.

Each square from (3.4) and (3.5) generates a corresponding braid of exact sequences similarly to that considered in Theorem 3.1.

Note that the left vertical maps in (3.4) and (3.5) induce maps of structure sets that geometrically are given by the restrictions of a normal map to the corresponding submanifold. 
Proof. We have the following homotopy commutative diagram of spectra

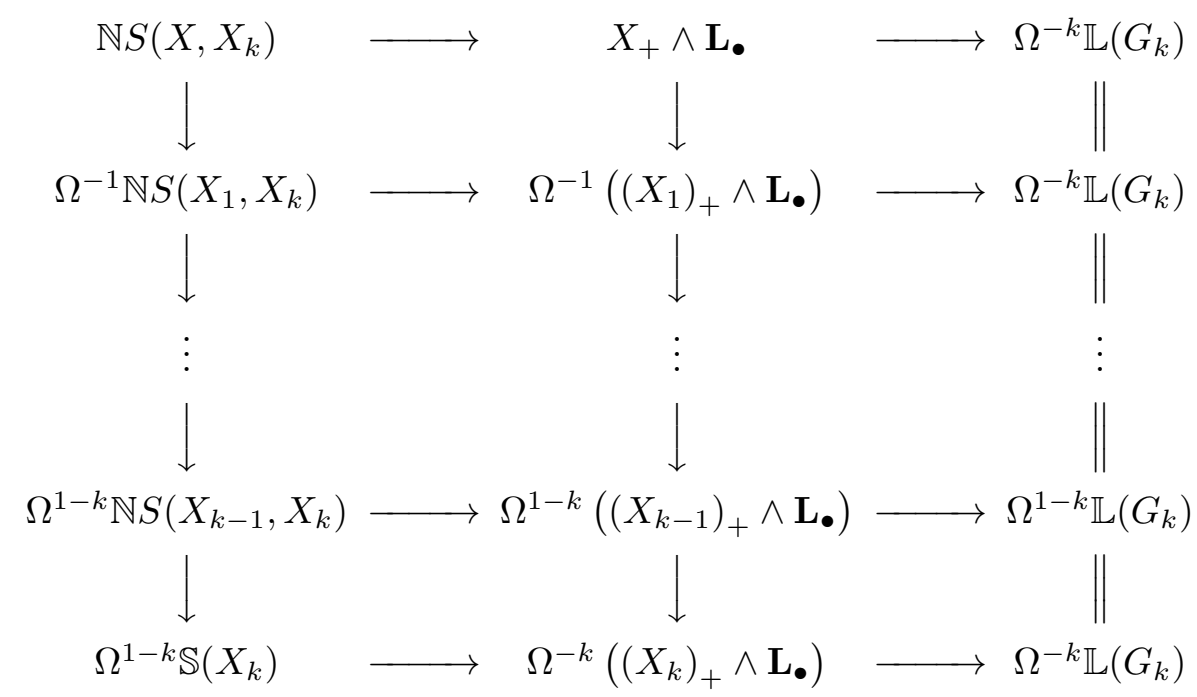

in which all right squares are pullback. Diagram (3.6) follows if we join together pullback squares (32) from [2] for all triples of manifolds $X_{k} \subset X_{i} \subset X_{i-1}$, where $k-1 \geq i \geq 1$, for the filtration $\mathcal{X}$.

Let a Browder-Livesay filtration $\mathcal{X}$ be given by a triple of manifolds $Z \subset Y \subset X$ [18]. Sometimes we shall denote this filtration by $(X, Y, Z)$. Denote by $F_{Z}$ the square of fundamental groups in the splitting problem (relative to the boundary) for $(Y \backslash Z, \partial(Y \backslash Z)) \subset(X \backslash Z, \partial(X \backslash Z))$.

Theorem 3.3. There exists a pullback square of spectra

$$
\begin{array}{ccc}
\mathbb{L}^{B Q}(X, Y, Z) & \rightarrow & \mathbb{L}\left(\pi_{1}(Z)\right) \\
\downarrow & & \downarrow \\
\Omega^{2} \mathbb{S}(X, Y, Z) & \rightarrow & \Omega \mathbb{N} S(X, Z)
\end{array}
$$

in which the fibres of the vertical maps are $X_{+} \wedge \mathbf{L}_{\bullet}$ and the cofibres of the horizontal maps are $\mathbb{L} P\left(F_{Z}\right)$.

Square (3.7) defines the braid of exact sequences

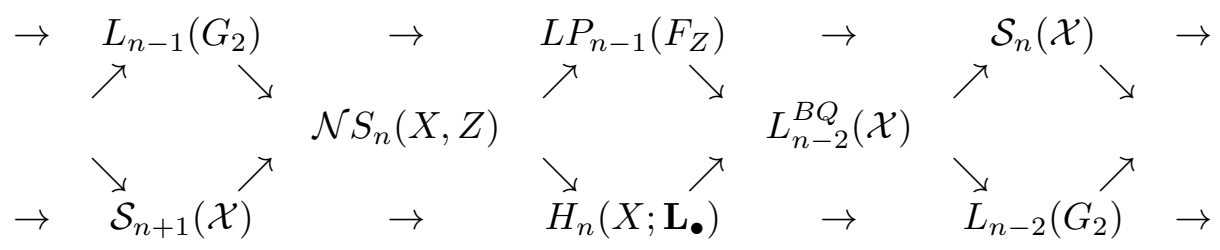

where $G_{2}=\pi_{1}(Z)$.

Proof. Consider a homotopy commutative diagram

$$
\begin{array}{cccc}
\Omega^{-1} \mathbb{L}^{B Q}(\mathcal{X}) & \rightarrow & \Omega^{-1} \mathbb{L}\left(\pi_{1}(Z)\right) \\
\downarrow & & \downarrow \\
\Omega \mathbb{S}(\mathcal{X}) & \rightarrow & \mathbb{N} S(X, Z) \\
\downarrow & & \\
X_{+} \wedge \mathbf{L} \bullet & & \rightrightarrows & X_{+} \wedge \mathbf{L} \bullet
\end{array}
$$

in which the upper map is the map from (2.7) with a cofiber $\Omega^{-1} \mathbb{L}^{B Q}(\overline{\mathcal{X}})$. For any filtration $\mathcal{X}=(X, Y, Z)$ we have $\mathbb{L}^{B Q}(\overline{\mathcal{X}})=\mathbb{L} P\left(F_{Z}\right)$. Now the proof is similar to that of Theorem 3.1. 
Theorem 3.4. Under the above assumptions, there exists a pullback square of spectra

$$
\begin{array}{ccc}
\Omega^{-1} \mathbb{L} P\left(F_{Z}\right) & \rightarrow & \mathbb{S}(\mathcal{X}) \\
\downarrow & & \downarrow \\
\mathbb{L}\left(\pi_{1}(X \backslash Z)\right) & \rightarrow & \mathbb{S}(X, Z)
\end{array}
$$

in which the fibres of the vertical maps are $\Omega^{-1} \mathbb{L} S\left(F_{Z}\right)$ and the fibres of the horizontal maps are $\mathbb{N} S(X, Z)$.

Square (3.9) defines the braid of exact sequences

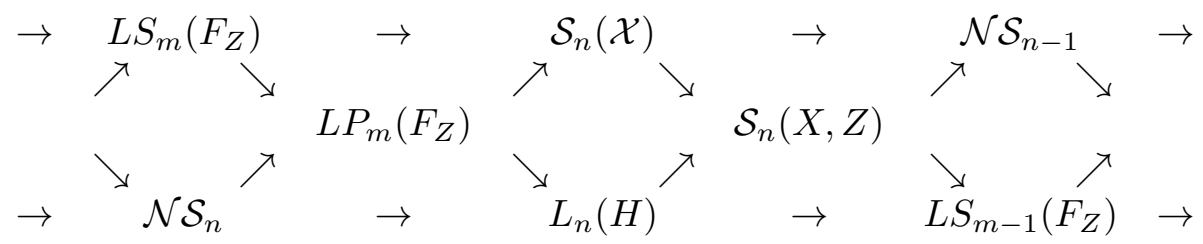

where $m=n-1, H=\pi_{1}(X \backslash Z)$, and $\mathcal{N S}_{*}=\mathcal{N S}_{*}(X, Z)$.

Proof. We have the natural maps of spectra (see [2], Theorem 6, and [13], Theorem $3.3)$ :

$$
\mathbb{S}(X, Y, Z) \rightarrow \mathbb{S}(X, Z) \rightarrow \Omega^{-1} \mathbb{N} S(X, Z) .
$$

This gives a homotopy commutative square of spectra

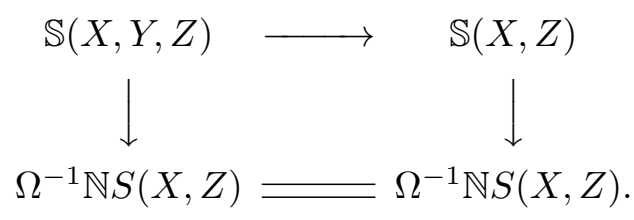

In (3.12) the fiber of the upper horizontal map is $\Omega^{-1} \mathbb{L} S\left(F_{Z}\right)$, the fiber of the left vertical map is $\Omega^{-1} \mathbb{L} P\left(F_{Z}\right)$ and the fiber of the right vertical map is $\mathbb{L}\left(\pi_{1}(X \backslash Z)\right)$ by [2] and [13]. Now we can complete the proof similarly to that of Theorem 3.1.

Theorem 3.5. The spectra of the structure sets of a Browder-Livesay filtration $\mathcal{X}=(X, Y, Z)$ fit into a bi-infinite homotopy commutative diagram of spectra

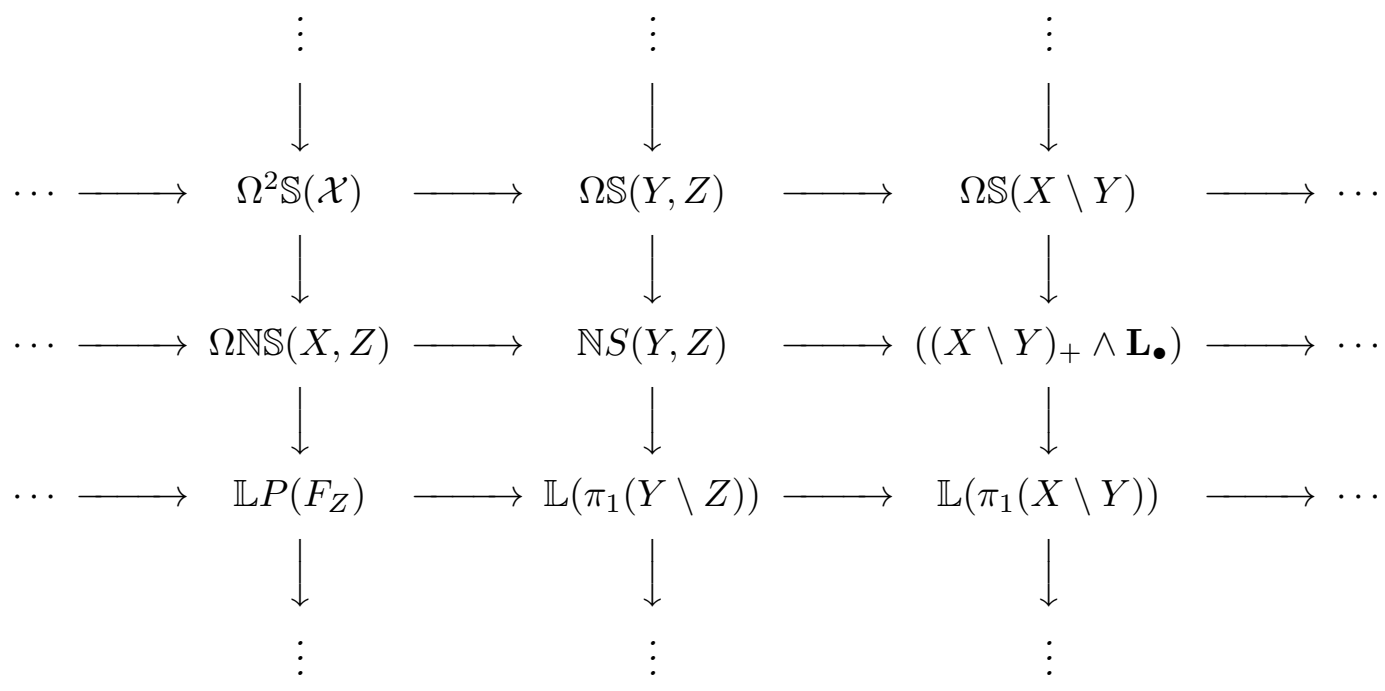


in which all rows and columns are cofibration sequences. Diagram (3.13) induces a bi-infinite commutative diagram of groups

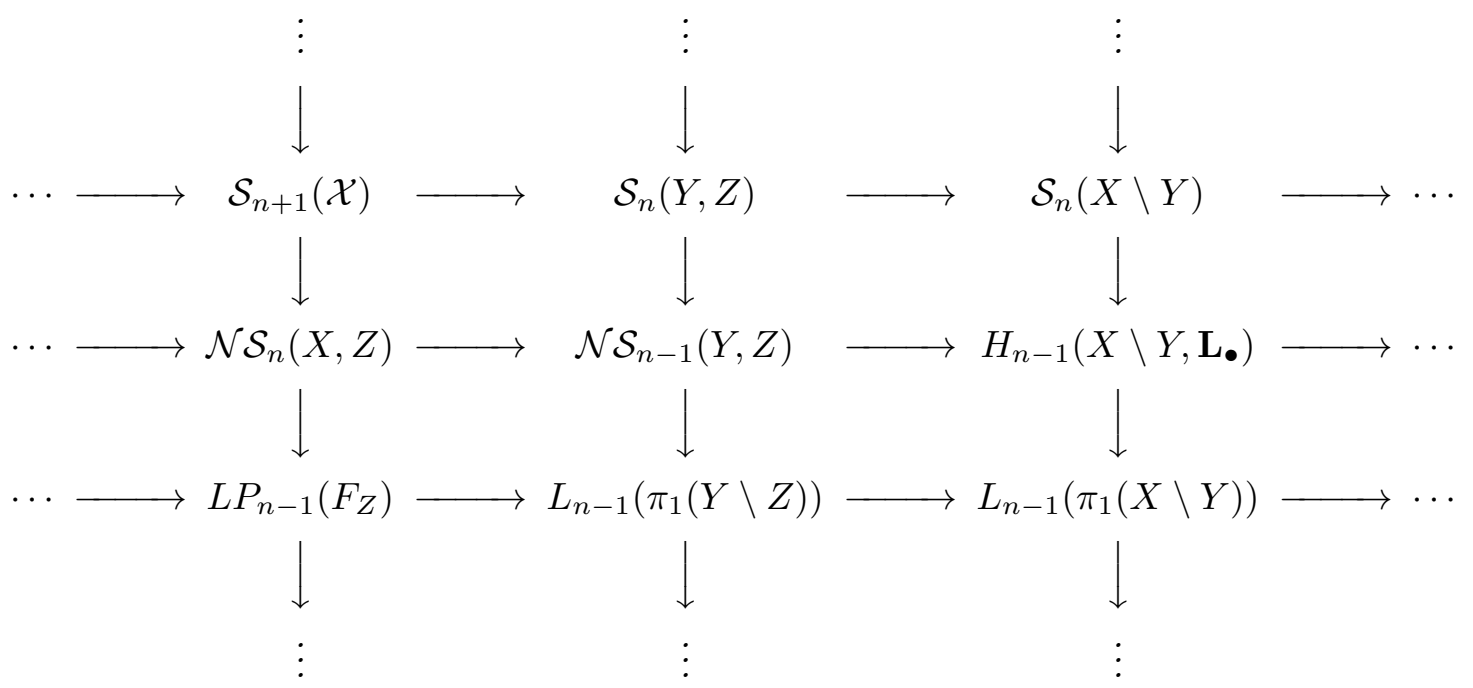

where all rows and columns are exact sequences.

Proof. The homotopy commutative left corner square in (3.13) is obtained by natural forgetful maps. The cofibers of the maps from this square are described in Theorem 3.3 of [13] and [2]. By [1], [15], and [21] we can extend this square by a bi-infinite homotopy commutative diagram of spectra (3.13). To obtain (3.14) it is sufficient to apply $\pi_{0}$ to the homotopy commutative diagram of spectra in (3.13).

Note that Theorems 3.3, 3.4, and 3.5 hold, in particular, for any triple of manifolds fitting into a filtration $\mathcal{X}$.

Theorem 3.6. For a Browder-Livesay filtration $\mathcal{X}$ as in (1.8) there is a pullback square of spectra

$$
\begin{array}{cccc}
\Omega^{-k+1} \mathbb{L}^{B Q}(\overline{\mathcal{X}}) & \rightarrow & \mathbb{S}(\mathcal{X}) \\
\downarrow & & \downarrow \\
\mathbb{L}\left(\pi_{1}\left(X \backslash X_{k}\right)\right) & \rightarrow & S\left(X, X_{k}\right)
\end{array}
$$

in which the fibres of the vertical maps are $\Omega^{-k+1} \mathbb{L} S F(\overline{\mathcal{X}})$ and the fibres of the horizontal maps are $\mathbb{N} S(X, Z)$.

Square (3.15) defines the braid of exact sequences

$$
\begin{aligned}
& \rightarrow \quad L S F_{m}(\overline{\mathcal{X}}) \quad \rightarrow \quad \mathcal{S}_{n}(\mathcal{X}) \quad \rightarrow \quad \mathcal{N S}_{n-1}\left(X, X_{k}\right) \\
& \nearrow \searrow \\
& L_{m}^{B Q}(\overline{\mathcal{X}}) \\
& \nearrow^{\mathcal{S}_{n}(\mathcal{X})} \\
& \stackrel{\searrow}{\mathcal{N S}_{n}\left(X, X_{k}\right)} \rightarrow \underset{L_{n}\left(\pi_{1}\left(X \backslash X_{k}\right)\right)}{\searrow} \\
& \mathcal{S}_{n}\left(X, X_{k}\right)
\end{aligned}
$$

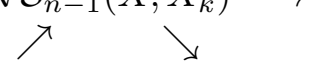

$$
\begin{aligned}
& \rightarrow \quad \underset{L}{L S} F_{m-1}(\overline{\mathcal{X}}) \quad \rightarrow
\end{aligned}
$$

where $m=n-k+1$.

Proof. Consider a homotopy commutative diagram

$$
\begin{aligned}
& \Omega^{-k+1} \mathbb{L}^{B Q}(\overline{\mathcal{X}}) \rightarrow \Omega^{-k} \mathbb{L}^{B Q}(\mathcal{X}) \quad \rightarrow \quad \mathbb{S}(\mathcal{X}) \\
& \downarrow \quad \downarrow \quad \downarrow \quad \downarrow \\
& \mathbb{L}\left(\pi_{1}\left(X \backslash X_{k}\right)\right) \rightarrow \Omega^{-k} \mathbb{L} P\left(\pi_{1}\left(X \backslash X_{k}\right)\right) \rightarrow \mathbb{S}\left(X, X_{k}\right)
\end{aligned}
$$


where the vertical maps correspond to passing from $\mathcal{X}$ to the subfiltration $X_{k} \subset X$, and the horizontal maps follow from (2.7) and stratified surgery exact sequence. The fiber of the bottom composition in (3.17) is $\mathbb{N} S\left(X, X_{k}\right)$ and the same is the fiber of the upper composition. This follows from the commutative diagram

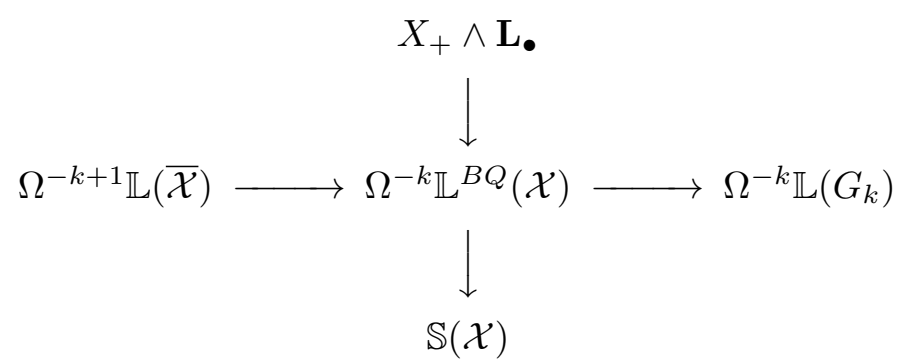

in which the row and the column are cofibrations. Hence the fibers of the compositions

$$
X_{+} \wedge \mathbf{L} \bullet \rightarrow \Omega^{-k} \mathbb{L}\left(G_{k}\right)
$$

and

$$
\Omega^{-k+1} \mathbb{L}(\overline{\mathcal{X}}) \rightarrow \mathbb{S}(\mathcal{X})
$$

coincide [15]. But the first fiber is $\mathbb{N} S\left(X, X_{k}\right)$ by [2] (see also Theorem 3.1). Now the result follows by standard arguments.

Proposition 3.7. Let $\mathcal{X}$ be a Browder-Livesay filtration as in (1.8), and $\mathcal{Y}_{j}$ a subfiltration

$$
X_{j} \subset X_{k-1} \subset \cdots \subset X_{1} .
$$

For $1 \leq j \leq k$ there is the following homotopy commutative diagram of spectra

$$
\begin{aligned}
& \mathbb{L}\left(G_{j}\right) \quad \rightarrow \quad \mathbb{L}^{B Q}\left(\overline{\mathcal{X}_{j}}\right) \rightarrow \Omega^{-1} \mathbb{L}^{B Q}\left(X_{j}\right)
\end{aligned}
$$

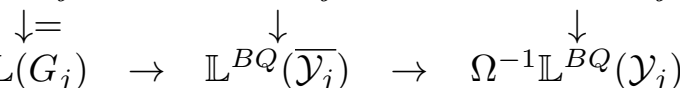

where the rows are cofibrations and the right square is a pullback. The cofiber of both right vertical maps is $\Omega^{j-1} \mathbb{L}\left(\pi_{1}\left(X_{0} \backslash X_{1}\right)\right)$.

Proof. The diagram in (3.18) follows from (2.7) by functoriality (see [19] and [26]). The right square is a pullback. Now the result follows from (2.31) in [3].

Theorem 3.8. Under the assumptions of Proposition 3.7, let $\mathcal{Y}=\mathcal{Y}_{k}$. We have a 
bi-infinite commutative diagram of groups

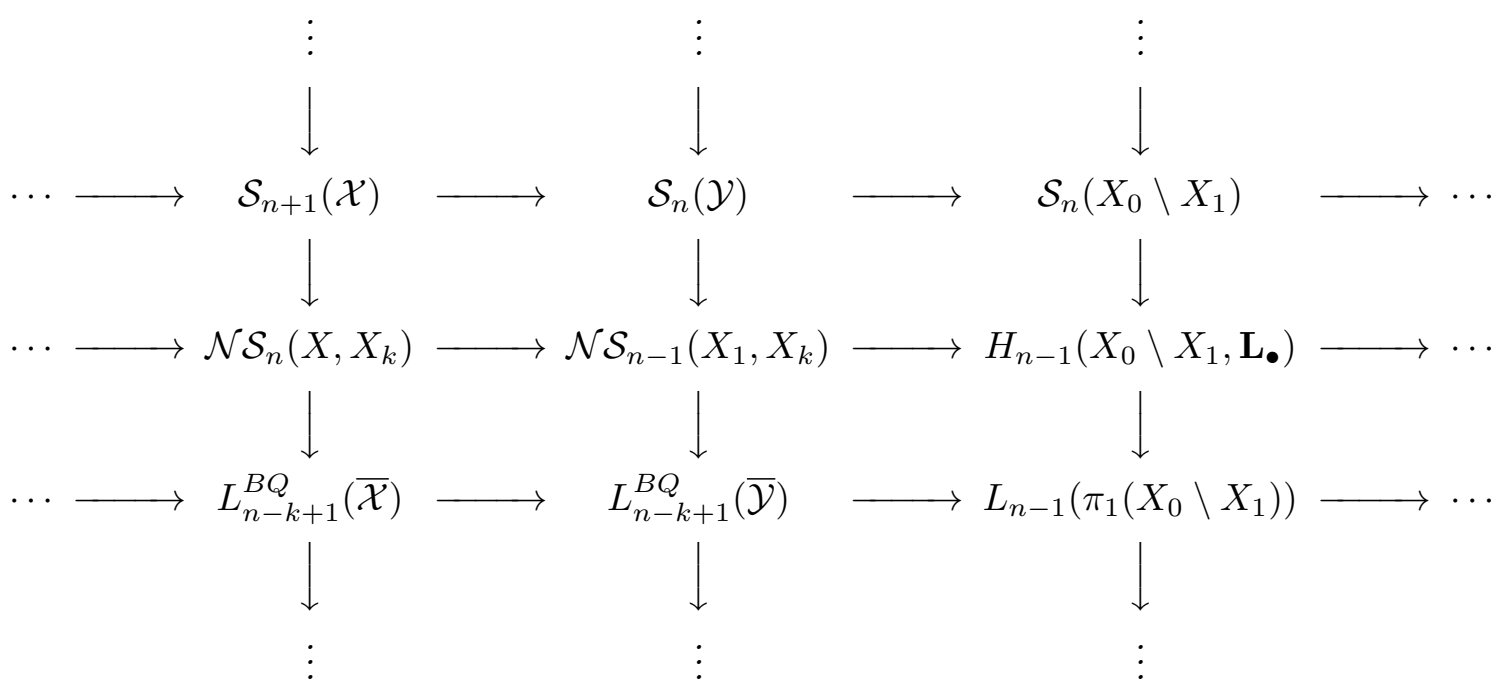

where all rows and columns are exact sequences.

Proof. Each of the first two columns follows from Theorem 3.6 for the filtrations $\mathcal{X}$ and $\mathcal{Y}=\mathcal{Y}_{k}$. The maps of the first two columns are induced by natural forgetful maps. The middle horizontal sequence is given in [2] and the upper horizontal sequence is given in (3.3) of [13]. Then the result follows similarly as above.

\section{Examples and Applications.}

In this section we illustrate several examples and applications of the obtained results. We maintain all notations from the previous sections.

Proposition 4.1. Let $\mathcal{X}$ be a Browder-Livesay filtration as in (1.8) such that $k \geq$ $3, \operatorname{dim} X=m, m-k \geq 5$, and $\pi_{1}(X)$ is an elementary 2-group with a nontrivial orientation of rank $r \geq 1$. Then for $m$ odd, we have an epimorphism

$$
\mathcal{S}_{m+1}\left(X, X_{k}\right) \longrightarrow \mathcal{N} \mathcal{S}_{m}\left(X, X_{k}, \xi\right)
$$

and for $m$ even we have an exact sequence

$$
\mathcal{S}_{m+1}\left(X, X_{k}\right) \longrightarrow \mathcal{N S}_{m}\left(X, X_{k}, \xi\right) \longrightarrow \mathbb{Z} / 2 \longrightarrow 0,
$$

where $\xi$ is a topological normal block bundle of $X_{k}$ in $X$.

Proof. For the pair $\left(X, X_{k}, \xi\right)$ the commutative diagram from [2, Theorem 3] has the following form

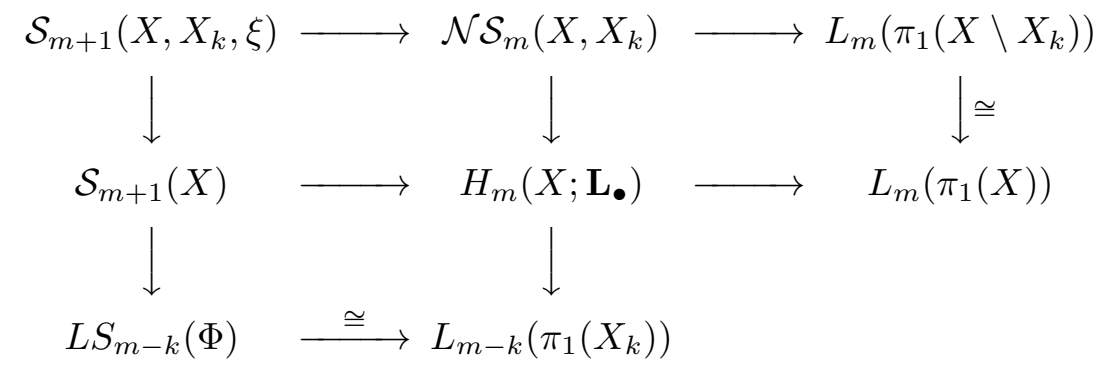


since the codimension $k$ of $X_{k}$ in $X$ is greater than 2. The map

$$
\mathcal{N S}_{m}\left(X, X_{k}\right) \rightarrow L_{m}\left(\pi_{1}\left(X \backslash X_{k}\right)\right)
$$

is trivial for $m$ odd and has image $\mathbb{Z} / 2$ for $m$ even. In fact, the map

$$
H_{m}\left(X, \mathbf{L}_{\bullet}\right) \rightarrow L_{m}\left(\pi_{1}(X)\right)
$$

is trivial for $m$ odd and has image $\mathbb{Z} / 2$ for $m$ even (see, for example, Theorem 3.4 from [11] (see also [12]). Now the statement is proved.

Proposition 4.2. Let $\mathcal{X}$ be a Browder-Livesay filtration as in (1.8) such that $n=$ $4 l+1$ and $\pi_{1}(X)$ an elementary abelian 2-group with trivial orientation of rank $r \geq 1$. For $k \geq i \geq 3$ and $n-i \geq 5$ there are epimorphisms

$$
\mathcal{S}^{s}\left(X, X_{i}\right) \longrightarrow \mathcal{N S}^{s}\left(X, X_{i}\right) \text {. }
$$

Proof. Using a commutative diagram similar to (4.3), it follows that the map

$$
\mathcal{N} \mathcal{S}_{4 l+1}\left(X, X_{i}\right) \rightarrow L_{4 l+1}\left(\pi_{1}\left(X \backslash X_{i}\right)\right)
$$

is trivial since the Assembly map $[X, G / T O P] \rightarrow L_{4 l+1}\left(\pi_{1}(X)\right)$ is trivial (see [12] and Theorem 3.4 from [11]). The same arguments used before allow us to complete the proof.

Now we compute various structure sets for a Browder-Livesay filtration $\mathcal{X}$

$$
\mathbb{R} P^{n-k} \subset \mathbb{R} P^{n-k+1} \subset \cdots \subset \mathbb{R} P^{n-1} \subset \mathbb{R} P^{n}
$$

given by a natural filtration of real projective space $\mathbb{R} P^{n}$ with $n-k \geq 5$. We recall here well-known results about some structure sets for the filtration $\mathcal{X}$ in (4.6) from [4], [14], and [22]:

$$
\mathcal{T}\left(\mathbb{R} P^{2 i+5}\right)=\mathcal{T}\left(\mathbb{R} P^{2 i+4}\right)=\bigoplus_{1}^{i+2} \mathbb{Z}_{2}
$$

that is,

$$
\begin{array}{cc}
\mathcal{T}\left(\mathbb{R} P^{4 k}\right)=\bigoplus_{1}^{2 k} \mathbb{Z}_{2}, & \mathcal{S}^{s}\left(\mathbb{R} P^{4 k}\right)=\bigoplus_{1}^{2 k-1} \mathbb{Z}_{2}, \\
\mathcal{T}\left(\mathbb{R} P^{4 k+1}\right)=\bigoplus_{1}^{2 k} \mathbb{Z}_{2}, & \mathcal{S}^{s}\left(\mathbb{R} P^{4 k+1}\right)=\bigoplus_{1}^{2 k} \mathbb{Z}_{2}, \\
\mathcal{T}\left(\mathbb{R} P^{4 k+2}\right)=\bigoplus_{1}^{2 k+1} \mathbb{Z}_{2}, & \mathcal{S}^{s}\left(\mathbb{R} P^{4 k+2}\right)=\bigoplus_{1}^{2 k} \mathbb{Z}_{2}, \\
\mathcal{T}\left(\mathbb{R} P^{4 k+3}\right)=\bigoplus_{1}^{2 k+1} \mathbb{Z}_{2}, & \mathcal{S}^{s}\left(\mathbb{R} P^{4 k+3}\right)=\bigoplus_{1}^{2 k} \mathbb{Z}_{2} \oplus \mathbb{Z},
\end{array}
$$

since these structure sets fit into the following surgery exact sequences

$$
\begin{gathered}
0=L_{1}\left(\mathbb{Z}_{2}^{-}\right) \rightarrow \mathcal{S}^{s}\left(\mathbb{R} P^{4 k}\right) \rightarrow \mathcal{T}\left(\mathbb{R} P^{4 k}\right) \stackrel{e p i}{\rightarrow} L_{0}\left(\mathbb{Z}_{2}^{-}\right)=\mathbb{Z}_{2}, \\
\mathbb{Z}_{2}=L_{2}\left(\mathbb{Z}_{2}\right) \stackrel{0}{\rightarrow} \mathcal{S}^{s}\left(\mathbb{R} P^{4 k+1}\right) \stackrel{\cong}{\rightarrow} \mathcal{T}\left(\mathbb{R} P^{4 k+1}\right) \rightarrow L_{1}\left(\mathbb{Z}_{2}\right)=0, \\
0=L_{3}\left(\mathbb{Z}_{2}^{-}\right) \rightarrow \mathcal{S}^{s}\left(\mathbb{R} P^{4 k+2}\right) \rightarrow \mathcal{T}\left(\mathbb{R} P^{4 k+2}\right) \stackrel{\text { epi }}{\rightarrow} L_{2}\left(\mathbb{Z}_{2}^{-}\right)=\mathbb{Z}_{2}, \\
\mathbb{Z}^{2}=L_{0}\left(\mathbb{Z}_{2}\right) \stackrel{\text { Im= }}{\rightarrow} \mathcal{S}^{s}\left(\mathbb{R} P^{4 k+3}\right) \rightarrow \mathcal{T}\left(\mathbb{R} P^{4 k+3}\right) \stackrel{\text { epi }}{\rightarrow} L_{3}\left(\mathbb{Z}_{2}\right)=\mathbb{Z}_{2} .
\end{gathered}
$$

From Proposition 7.2.6 ii) of [20] we obtain the exact sequence

$$
\cdots \rightarrow \mathcal{S}_{m}\left(\mathbb{R} P^{n} \backslash \mathbb{R} P^{n-1}\right) \rightarrow \mathcal{S}_{m}\left(\mathbb{R} P^{n}, \mathbb{R} P^{n-1}, \xi\right) \rightarrow \mathcal{S}_{m-1}\left(\mathbb{R} P^{n-1}\right) \rightarrow \ldots
$$

The manifold $\mathbb{R} P^{n} \backslash \mathbb{R} P^{n-1}$ is topologically an open disk $D^{n}$. Hence we have

$$
\mathcal{S}_{m}\left(\mathbb{R} P^{n} \backslash \mathbb{R} P^{n-1}\right)=0 \text { for all } n,
$$

$$
H_{n}\left(\mathbb{R} P^{n} \backslash \mathbb{R} P^{n-1} ; \mathbf{L}_{\bullet}\right)=L_{n}(1)=\mathbb{Z}, 0, \mathbb{Z}_{2}, \quad 0 \text { for } n=0,1,2,3 \bmod 4
$$

(see [19], p.276). From (4.10) and (4.11) we obtain isomorphisms

$$
\mathcal{S}^{s}\left(\mathbb{R} P^{n}, \mathbb{R} P^{n-1}, \xi\right) \cong \mathcal{S}^{s}\left(\mathbb{R} P^{n-1}\right) .
$$


Theorem 4.3. Let $\mathcal{X}=\mathcal{X}_{n}$ be a filtration

$$
\mathbb{R} P^{k} \subset \mathbb{R} P^{k+1} \subset \cdots \subset \mathbb{R} P^{n-1} \subset \mathbb{R} P^{n}
$$

where $5 \leq k<n$, and $\mathcal{X}_{m}$ a subfiltration

$$
\mathbb{R} P^{k} \subset \mathbb{R} P^{k+1} \subset \cdots \subset \mathbb{R} P^{n-1} \subset \mathbb{R} P^{m}
$$

where $k \leq m<n$. Then we have isomorphisms

$$
\mathcal{S}^{s}(\mathcal{X}) \cong \mathcal{S}^{s}\left(\mathcal{X}^{m}\right) \cong \mathcal{S}^{s}\left(\mathbb{R} P^{k}\right)
$$

where the structure sets $\mathcal{S}^{s}\left(\mathbb{R} P^{k}\right)$ are given in (4.8).

Proof. We use the upper horizontal exact sequence in (3.19). An induction argument on the number of manifolds in the filtration and the isomorphism $\mathcal{S}_{*}\left(\mathbb{R} P^{i} \backslash\right.$ $\left.\mathbb{R} P^{i-1}\right)=0$ in (4.11) provide the result.

Theorem 4.4. Let $\mathbb{R} P^{n}$ be the real projective space of dimension $n \geq 6$ with the subspace $\mathbb{R} P^{n-1}$ of codimension $q=1$. Then we have:

i)

$$
\mathcal{N} \mathcal{S}^{s}\left(\mathbb{R} P^{n}, \mathbb{R} P^{n-1}\right) \cong \mathcal{S}^{s}\left(\mathbb{R} P^{n}, \mathbb{R} P^{n-1}, \xi\right) \cong \begin{cases}\bigoplus_{j=1}^{2 k-1} \mathbb{Z}_{2}, & \text { if } n=4 k+1 \\ \bigoplus_{j=1}^{2 k} \mathbb{Z}_{2}, & \text { if } n=4 k+3\end{cases}
$$

ii) For $n=4 k+2, \mathcal{N} \mathcal{S}^{s}\left(\mathbb{R} P^{n}, \mathbb{R} P^{n-1}\right) \cong \mathcal{T}\left(\mathbb{R} P^{4 k+2}\right)=\bigoplus_{j=1}^{2 k+1} \mathbb{Z}_{2}$.

iii) For $n=4 k, \mathcal{N S}^{s}\left(\mathbb{R} P^{4 k}, \mathbb{R} P^{4 k-1}\right) \cong \mathcal{S}^{s}\left(\mathbb{R} P^{4 k-1}\right) \oplus \mathbb{Z}$.

Proof. If $X=\mathbb{R} P^{n}$ and $Y=\mathbb{R} P^{n-1}$, then $X \backslash Y=D^{n}$ and $\pi_{1}\left(D^{n}\right)=1$.

In case i) for $n=4 k+1$, let us consider the part of commutative diagram (23) from $[2]$ :

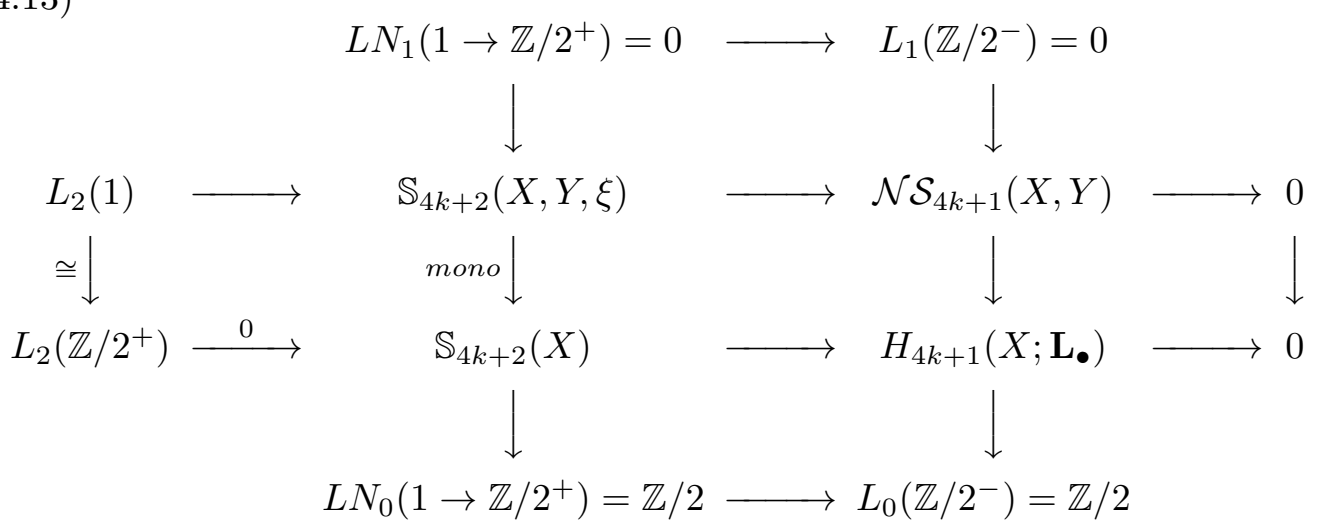

where $L N_{i}\left(1 \rightarrow \mathbb{Z} / 2^{+}\right)=L S_{i}\left(F^{+}\right)$. Here $F^{ \pm}$is a square of fundamental groups in the splitting problem for the pair $(X, Y)=\left(\mathbb{R} P^{n}, \mathbb{R} P^{n-1}\right)$, where the orientation is + for $n$ odd and - otherwise. In (4.13) we use isomorphisms $L_{1}(1)=L_{1}\left(\mathbb{Z} / 2^{+}\right)=0$ and (4.9). Now the statement i) for $n=4 k+1$ follows. The case $n=4 k+3$ can be obtained using similar arguments. So case i) is completely proved. 
In case ii) the diagram, which is similar to that in (4.13), has the form

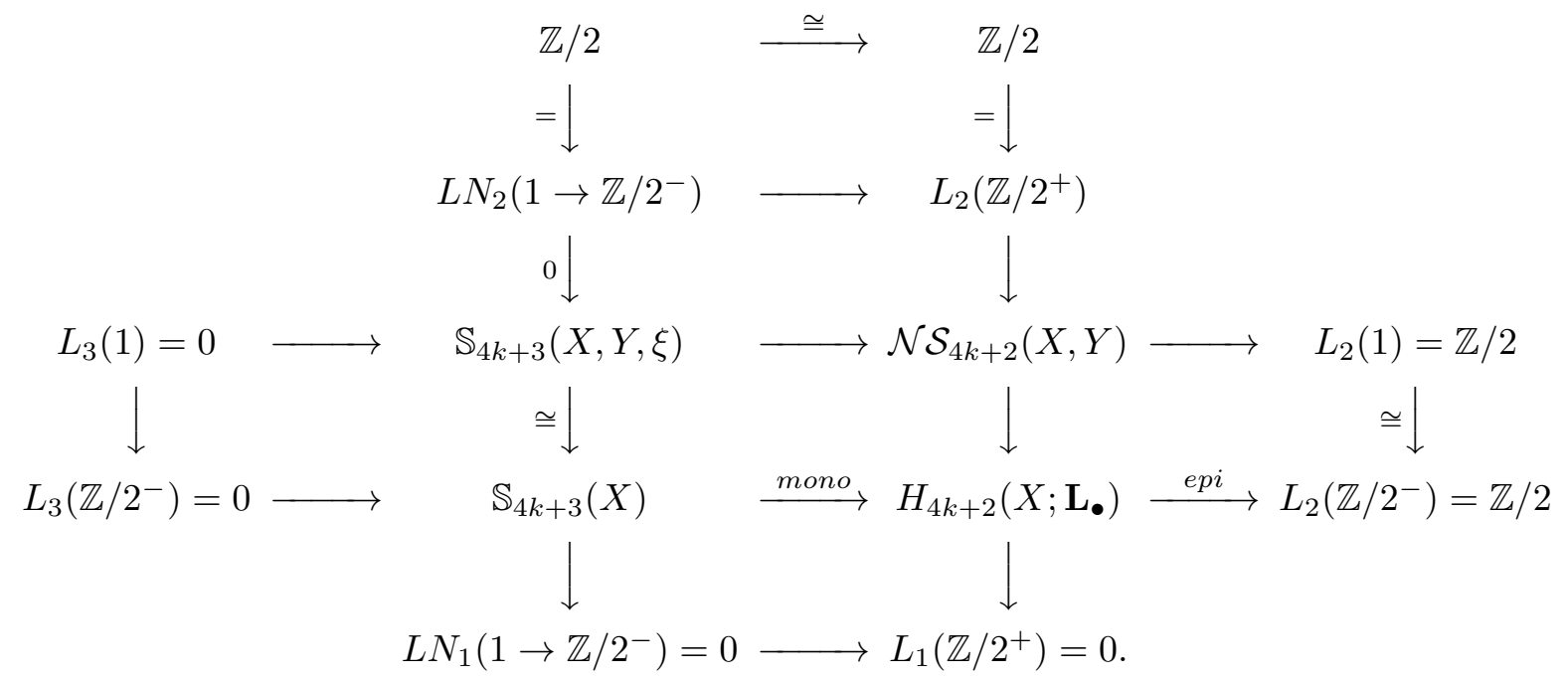

Here we use (4.8) and (4.9) and the computation of Wall groups in [23]. Now statement ii) follows.

In case iii) the diagram, which is similar to that in (4.13), has the form

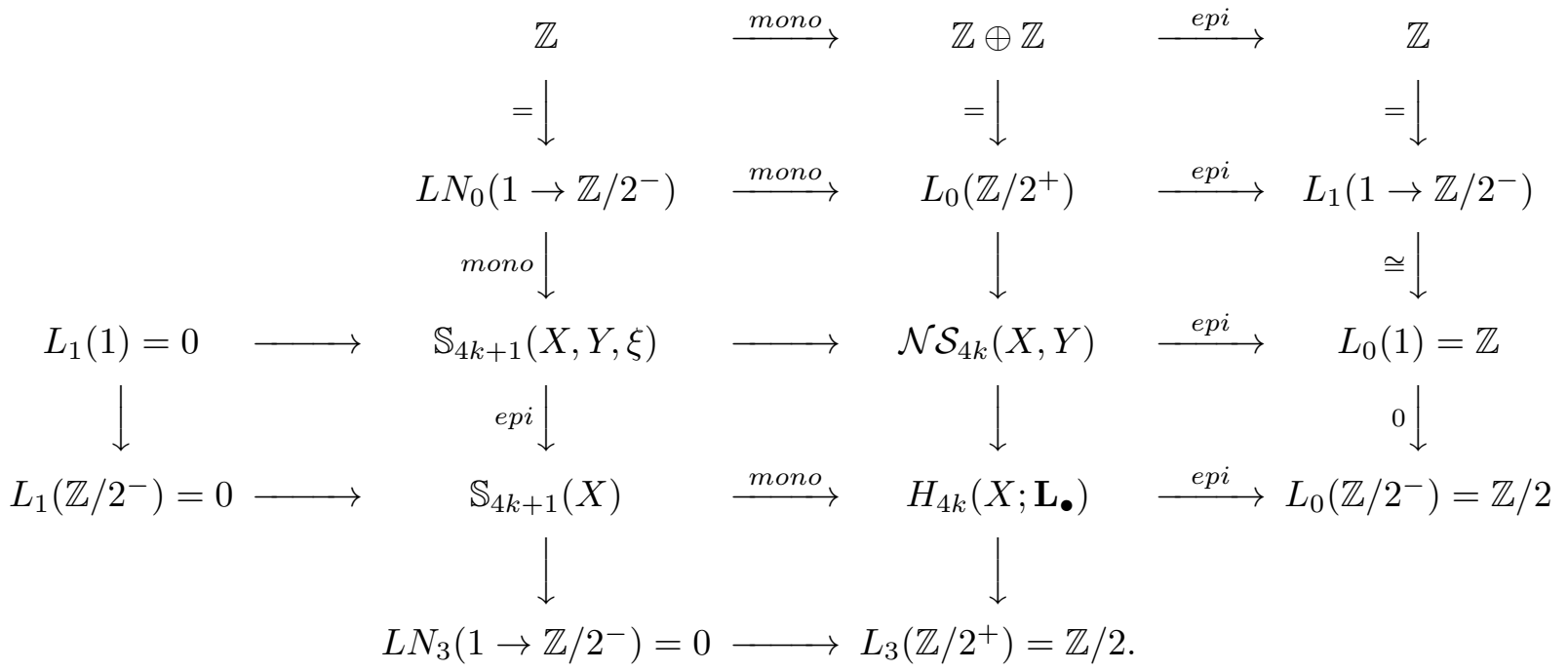

Here we use (4.8) and (4.9) and the computation of Wall groups in [23]. Now statement iii) follows by a diagram chasing. Thus the theorem is completely proved.

Acknowledgements. The first and fourth authors were partially supported by the GNSAGA of the National Research Council of Italy and by the MIUR (Ministero della Istruzione, Università e Ricerca) of Italy, and by a Research Grant of the University of Modena and Reggio Emilia. The third author was partially supported by CONACyT Grant 98697. 


\section{REFERENCES}

1. A. Bak - Yu. V. Muranov, Splitting along submanifolds and L-spectra, Journal of Math. Sciences 123 (4) (2004), 4169-4184.

2. A. Bak-Yu.V. Muranov, Normal invariants of manifold pairs and assembly maps, Sb.: Math. 197 (2006), 791-811; English transl. in Sbornik: Math. 197 (2006), 791-811.

3. A. Bak - Yu.V. Muranov, Splitting of a simple homotopy equivalence along a submanifold with filtration, Sb.: Math. 199 (2008), 3-26.

4. A. Bak - Yu.V. Muranov, Surgery on a pair of transversal manifolds, Journal of Homotopy and Related Structures (2012); i www.springerlink.com/ content/00p0j7j0p5531j81/i.

5. W. Browder - G. R. Livesay, Fixed point free involutions on homotopy spheres, Bull. Amer. Math. Soc. 73 (1967), 242-245.

6. W. Browder - F. Quinn, A surgery theory for G-manifolds and stratified sets, in ManifoldsTokyo 1973 (1975), Univ. of Tokyo Press, 27-36.

7. S. E. Cappell - J. L. Shaneson, Pseudo-free actions. I, Lect. Notes in Math. 763 (1979), 395-447.

8. A. Cavicchioli - Yu.V. Muranov - F. Spaggiari, Relative groups in surgery theory, Bull. Belg. Math. Soc. Simon Stevin 12 (2005), 109-135.

9. A. Cavicchioli- Yu.V. Muranov- F. Spaggiari, Mixed structures on a manifold with boundary, Glasgov Math. J. 48 (2006), 125-143.

10. I. Hambleton - A. Ranicki - L. Taylor, Round L-theory, J. Pure Appl. Algebra 47 (1987), 131-154.

11. F. Hegenbarth - Yu. V. Muranov, On the surgery assembly map, International Math. Forum 3 (2008), no. 5, 209-228.

12. A. F. Kharshiladze, Surgery on manifolds with finite fundamental groups, Uspechi Mat. Nauk 42 (1987), 55-85; English transl. in Russian Math. Surveys 42 (1987).

13. R. Jimenez - Yu. V. Muranov, Structure sets of triples of manifolds, Journal of Math. Sciences 144 (2007), no. 5, 4468-4483.

14. S. Lopez de Medrano, Involutions on manifolds, Springer-Verlag, Berlin-Heidelberg-New York, 1971.

15. Y.V. Muranov, Splitting obstruction groups and quadratic extensions of anti-structures, Izvestiya: Mathematics 59:6 (1995), 1207-1232; English transl. from Izvestiya RAN: Ser. Mat. 59:6, $107-132$.

16. Yu V. Muranov, Splitting problem, Trudi MIRAN (in Russian) 212 (1996), 123-146; English transl. in Proc. of the Steklov Inst. of Math. 212 (1996), 115-137.

17. Yu. V. Muranov - D. Repovš - R. Jimenez, Surgery spectral sequence and manifolds with filtration, Trudy MMO 67 (2006), 294-325; English translation in: Trans. Moscow Math. Soc. 67 (2006), 261-288.

18. Yu. V. Muranov - D. Repovš - F. Spaggiari, Surgery on triples of manifolds, Mat. Sbornik 8 (2003), 139-160; English transl. in Sbornik Mathematics 194 (2003), 1251-1271.

19. A. A. Ranicki, The total surgery obstruction, Lecture Notes in Math. 763 (1979), 275-316.

20. A. A. Ranicki, Exact Sequences in the Algebraic Theory of Surgery, Math. Notes 26, Princeton Univ. Press, Princeton, N. J., 1981.

21. R. Switzer, Algebraic Topology-Homotopy and Homology, Grund. Math. Wiss. 212, SpringerVerlag, Berlin-Heidelberg-New York, 1975.

22. C. T. C. Wall, Surgery on Compact Manifolds, Academic Press, London - New York, 1970; Second Edition (A. A. Ranicki, ed.), Amer. Math. Soc., Providence, R.I., 1999.

23. C.T.C. Wall, Classification of Hermitian forms, VI. Group rings, Ann. of Math. (2) 103 (1976), 1-80.

24. S. Weinberger, The Topological Classification of Stratified Spaces, The University of Chicago Press, Chicago - London, 1994. 